\title{
FIRE DYNAMIC SIMULATION ON THERMAL ACTIONS IN LOCALIZED FIRES IN LARGE ENCLOSURE
}

\author{
Chao Zhang ${ }^{1, *}$ and Guo-qiang $\mathrm{Li}^{2}$ \\ ${ }^{1}$ College of Civil Engineering, Tongji University, 1239 Siping Road, Shanghai 200092, China \\ ${ }^{2}$ Sate Key Laboratory for Disaster Reduction in Civil Engineering, 1239 Siping Road, Shanghai, China \\ *(Corresponding author: E-mail: 08_chao_zhang@tongji.edu.cn)
}

Received: 11 April 2011; Revised: 14 July 2011; Accepted: 21 July 2011

\begin{abstract}
Large enclosures commonly exist in many buildings like atria, open car parks and airport terminals. There is an international trend to prompt performance-based method (PBM) for fire resistance design. By PBM, the temperatures of structures in design fires should be determined. Localized fires are always adopted as design fires in large spaces. Currently, no agreed calculation method is available for the calculation of the heat flux from a localized fire to a vertical column. This paper aims at providing a feasible way of calculating the thermal actions in localized fires. Particularly, gas temperatures in localized fires and heat transfer from localized fires to steel vertical columns have been numerically investigated. The popular CFD code FDS is adopted as the numerical tool. A design fire scenario and four real localized fire tests are simulated in FDS. The effects of input parameters, including grid size and number of solid angles, on the accuracies of the numerical results have been investigated. Numerical results are compared with correlations and test data, which shows good agreement.
\end{abstract}

Keywords: Fire Dynamic Simulation (FDS), Steel column, Localized fire, Heat transfer, Temperature calculation, Grid size

\section{INTRODUCTION}

A compartment fire will generally undergo six stages which include ignition, growth, flashover, full fire development or steady burning, decay and extinguishment. Flashover is the rapid transition between the primary fire which is essentially localized around the item first ignited, and the general conflagration within the compartment when all fuel surfaces are burning [1]. Depending on whether flashover will happen or not, the real fires are usually divided into pre- and post- flashover fires. For small and middle scaled compartments with sufficient fuel and ventilation, the potential fires will develop to flashover and be characterized as post-flashover fires. For large scale enclosures or where sprinklers work effectively, flashover is unlikely to occur and the fires are characterized as pre-flashover fires. Post-flashover fires provide the worst case scenarios which are usually considered in fire resistance design. However, localized heating of key elements of structure in pre-flashover fires must be also considered.

For post-flashover fires, the gas properties within the compartment are approximately uniform that temperature-time curves are usually adopted to represent the fire environments. Correspondingly, the temperature of exposed members can be easily determined by interpreting the homogenous gas temperature as the effective black body radiation temperature and as the same gas temperature for convection calculation. At present, various formulae are provided by fire codes in different countries for calculating the temperature of steel members in post-flashover fires [2-4]. However, for pre-flashover or localized fires, the distribution of gas temperature is spatially non-uniform. Although correlations had been derived for calculating the fire environment in localized fires (e.g. given by the Annex C in EC1 [5]), no agreed analytical method is available for calculating the temperature response of steel columns exposed to localized fires. In EC1 [5], correlations proposed by Hasemi are adopted to calculate the total heat fluxes from localized fires to horizontal members. Heat fluxes from fires (flames) to adjacent vertical walls have also been studied by many 
researchers, and calculation formulae are proposed in SFPE handbook [6]. To the authors' acknowledge, only Kamikawa et al. [7] had tested the thermal mechanism of steel columns exposed to localized fires.

Simulation method is a feasible way to predict the thermal action in localized fires. This paper is an extension of a previous work [8], concerning on using FDS [9] to simulate the heating mechanism of steel columns in localized fires. In [8], the surrounding fire test with heat release rate of $81 \mathrm{~kW}$ in [7] had been simulated by using FDS (where 'surrounding' is the location of the fire source to the column), and the numerical result agree well with the test data. To give general recommendations on using FDS for predicting the thermal actions in localized fires, the other 3 surrounding fire tests in [7] are simulated in this paper, and the results for gas temperatures in a design fire scenario predicted by FDS are compared with those predicted by correlations. Sensitivity studies have been conducted to investigate the effects of input parameters include grid size and number of solid angles, on the accuracies of the numerical results.

\section{CORRELATIONS FOR LOCALIZED FIRES}

\subsection{Plume Temperature}

A localized fire is loosely divided into the lower combustion (flame) and the upper non-combustion (plume) regions, as shown in Figure 1. In Figure 1, the ceiling jet, which refers to the relatively rapid gas flow in a shallow layer beneath the ceiling surface that is driven by the buoyancy of the hot combustion products from the plume, is also illustrated. Assuming the plume gases are ideal gases produced from a virtual point source which extend vertically due to buoyancy force, and assuming profiles of the distributions of the plume temperature and velocity, by solving the balance equations for mass, momentum and heat of plume gases, correlations for calculating the plume temperature had been derived by the classic plume theory [10]. The correlation derived from top-hat assumption [11] which assumes uniform distributions of plume temperature and velocity in horizontal plane is widely used in smoke control calculation, such as NFPA 92B, TM 119.

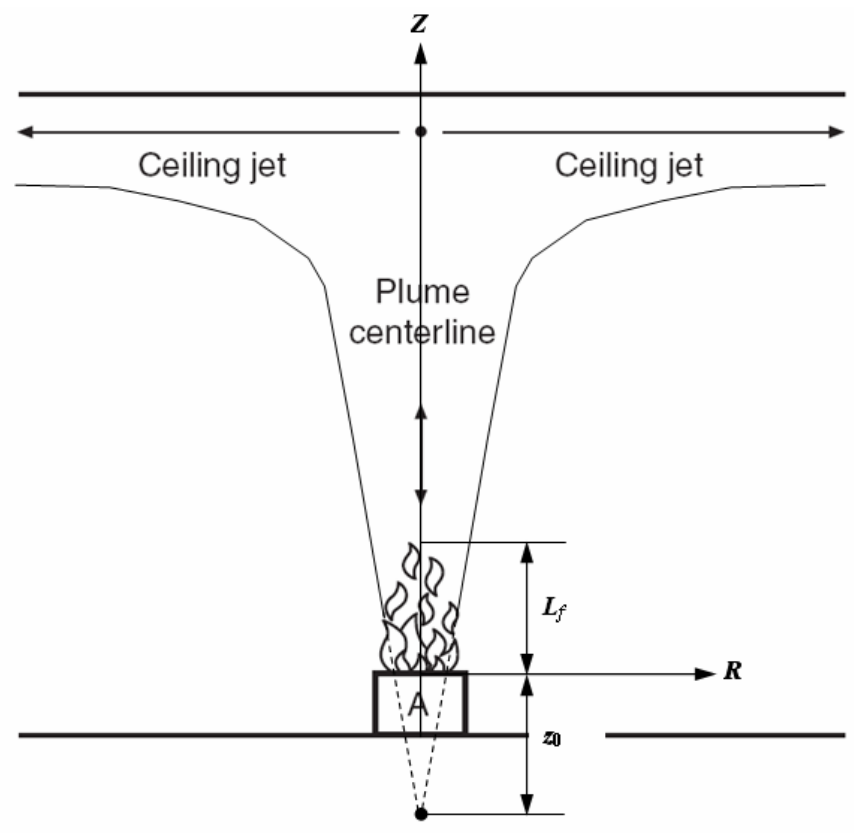

Figure 1. Illustration of the Localized Fire Model 
Assume profile of temperature rise is represented as Gaussian in shape, the plume temperature is calculated by [11]

$$
\Delta T=\Delta T_{0} \exp \left[-\left(\frac{R}{\sigma_{T}}\right)^{2}\right]
$$

with

$$
\sigma_{T}=1.20 b_{\Delta T}
$$

$$
b_{\Delta T}=0.12\left(\frac{T_{0}}{T_{\infty}}\right)^{1 / 2}\left(z-z_{0}\right)
$$

where $T_{0}$ is the plume centerline temperature calculated by

$$
\Delta T_{0}=9.1\left(\frac{T_{\infty}}{g c_{p}^{2} \rho_{\infty}^{2}}\right)^{1 / 3} \dot{Q}_{c}^{2 / 3}\left(z-z_{0}\right)^{-5 / 3}
$$

Taking the values of constant into Eq. 4, we get the correlation recommended in EC1 [5], thus

$$
T_{0}=20+0.25\left(1000 \dot{Q}_{c}\right)^{2 / 3}\left(z-z_{0}\right)^{-5 / 3}
$$

in which, $\dot{Q}_{c}=\left(1-\chi_{r}\right) \dot{Q}$ is the convective part of the heat release rate of the fire source; $\chi_{r}$ is the radiative fraction, and; $z_{0}$ is the height of the virtual source, calculated by

$$
z_{0}=-1.02 D+0.083 \dot{Q}^{2 / 5}
$$

here, $D=\sqrt{4 A / \pi}$ is the equivalent diameter of the fire, in which $A$ is the area of the fire source.

\subsection{Flame Temperature}

Eq. 4 is not valid for calculation in the flame region. The flame temperature can be calculated using the correlation given by Quintiere and Grove [12], thus

$$
\Delta T_{\text {flame }}=C_{T, f}\left(1-\chi_{r}\right) \frac{\Delta H_{c} / s_{a}}{c_{p}}
$$

where $C_{T, f}$ is a constant dependent on a fit to data, which is usually taken as $0.50[13] ; \Delta H_{c} / s_{a}$ is the heat released by consuming a unit mass of air, for most common fuel $\Delta H_{c} / s_{a}=3.03 \pm 0.02$ $\mathrm{kJ} / \mathrm{g}$ [1]; and $c_{p}$ is the specific heat of the gas, often taken as $1.00 \mathrm{~J} / \mathrm{gK}$ 。Correspondingly, Eq. (7) can be approximated by

$$
\Delta T_{\text {flame }}=1500\left(1-\chi_{r}\right)
$$

Eq. 7 or 8 assumes that all of the air entrainment into the flame zone is completely consumed. However, in practice, with height increase the amount of air entrainment will become more than that of air required for reaction and the exceeded air will cool the flame temperature. 
The flame and plume regions are usually divided by flame height. The correlation given by Heskestad for calculating the flame height is adopted by EC1 [5], which is given by

$$
L_{f}=-1.02 D+0.235 \dot{Q}^{2 / 5}
$$

\subsection{Ceiling Jet Temperature}

Correlations derived by Alpert [14] are widely used in designing smoke detectors and are recommended by PD 7974-2 [15] to determine the maximum temperature of the ceiling jets under an unconfined ceiling, which are given by

$$
\begin{aligned}
& \Delta T=16.9 \frac{\dot{Q}^{2 / 3}}{H^{5 / 3}} \quad(r / H \leq 0.18) \\
& \Delta T=5.38 \frac{\dot{Q}^{2 / 3} / H^{5 / 3}}{(r / H)^{2 / 3}} \quad(r / H>0.18)
\end{aligned}
$$

where $H$ is the height of the ceiling; and $r$ is the horizontal distance from the plume axis.

Alpert's correlations are derived for weak plume-driven ceiling jet flow, for strong plume-driven ceiling jet flow where the flame height of the fire plume is comparable to the height of the ceiling, correlation given by Heskestad and Hamada [16] is recommended for calculating the maximum temperature of the ceiling jet, thus

$$
\frac{\Delta T}{\Delta T_{p}}=1.92\left(\frac{r}{b}\right)^{-1}-\exp \left[1.61\left(1-\frac{r}{b}\right)\right] \quad\left(1 \leq \frac{r}{b} \leq 40\right)
$$

where $\Delta T_{p}$ is the excess temperature on the plume centerline at the level of the ceiling and $b$ is the characteristic plume radius given by

$$
b=0.045 \frac{T_{p}^{1 / 2} \dot{Q}_{c}^{2 / 5}}{\Delta T_{p}^{3 / 5}}
$$

\section{DESCRIPTION OF THE NUMERICAL TOOL}

The CFD code FDS [9] is adopted as the numerical tool. FDS is developed in particular for fire related simulations. In FDS, the fluid flow is modeled by solving the conservation equations for mass, mixture fraction, momentum and energy in a low Mach number form. The effect of the flow field turbulence is treated by means of the Smagorinsky form of Large-Eddy Simulation (LES), in which the large scale eddies are computed directly and sub-grid scale dissipative processes are modeled. An explicit, second-order accurate scheme is used for the time integration.

The combustion model in FDS is based on the assumption that the combustion is mixing-controlled. This implies that all species of interest can be described in terms of the mixture fraction $Z$, a conserved scalar variable. Heat from the reaction of fuel and oxygen is released along an infinitely thin sheet where $Z$ takes on its stoichiometric value as determined by the solution of the transport equation for $Z$. 
The radiative heat transfer is computed by solving the radiation transport equation (RTE) for a non-scattering grey gas using a Finite Volume Method (FVM) on the same grid as the flow solver. Solving the RTE over all wavelengths is computationally expensive; therefore, the radiation spectrum is divided into small bands. However, in most large-scale fires, soot is the most important combustion product controlling the thermal radiation. The gas can be described as a gray medium, such that the radiation spectrum of soot is continuous, reducing the number of spectral bands to a single band. The absorption coefficients are calculated using a narrow-band model called RadCal [17].To obtain the discretized form of the RTE, the unit sphere is divided into a finite number of the solid angles.

The governing equations are approximated on one or more rectilinear grids that obstructions with complex geometries are approximated with groups of prescribed rectangles in FDS. More and detail description of the mathematical models in FDS can be found in [9].

\section{DESIGN FIRE SCENARIO}

\subsection{Description}

Figure 2 shows the numerical model in FDS for the design fire scenario. The fire source is a $2.0 \mathrm{~m}$ square porous propane burner, which produces steady heat release rate of $1.6 \mathrm{MW}$ for 400 second. The area of the floor is $10 \mathrm{~m} \times 10 \mathrm{~m}=100 \mathrm{~m}^{2}$, and the height of the ceiling is $5 \mathrm{~m}$. The fire source is located at the centre of the floor. The design fire scenario can represent the small news agents or small shop fires in large enclosure [18].

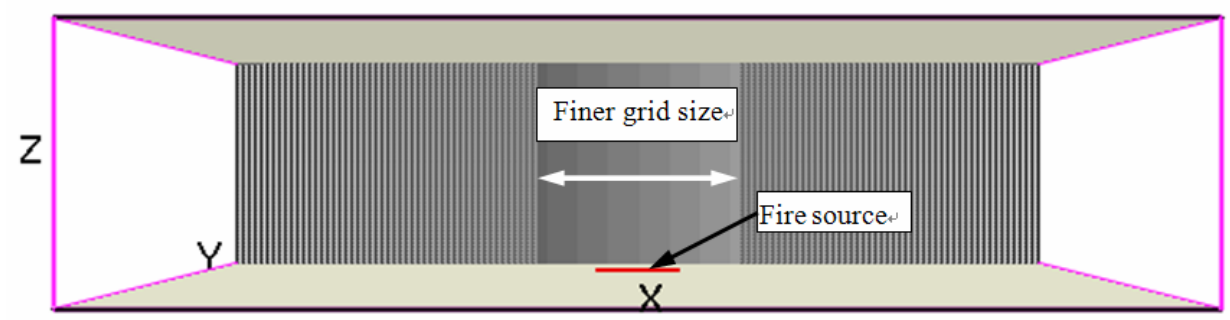

Figure 2. Numerical Model for the Design Fire Scenario

\subsection{Grid Sizes}

The grid sizes used is one of the most important numerical parameter in CFD dictating its numerical accuracy. The necessary spatial resolution for a proper LES simulation is customary defined in terms of the characteristic diameter of a plume, which is defined as [9],

$D^{*}=\left(\frac{\dot{Q}}{\rho_{\infty} c_{p} T_{\infty} \sqrt{g}}\right)^{2 / 5}$

The special resolution $R^{*}$ of a numerical grid is defined as,

$R^{*}=\frac{\delta x}{D^{*}}$ 
where $\delta x$ is the characteristic length of a cell for a given grid. The necessary resolution suggested in most studies is between 1/5 and 1/20 [9]. Ma and Quintiere [13] suggested resolution of 1/20 to successfully predict the flame height. Resolution between $1 / 10$ and $1 / 15$ were chosen by Gutierrez-Montes et al. [19] to successfully model atrium fires. Lin et al. [20] suggested $R^{*}=1 / 13$ is enough for CFD simulations to resolve the fire characteristics (flame height and thermal radiation).

For the design fire scenario, both coarse and fine grid sizes are used. For the coarse case, uniform grid size of $0.1 \mathrm{~m}\left(R^{*}=1 / 10\right)$ in XYZ directions are used, and the computation domain is consisted of 2,000,000 control volumes. For the fine case, near the fire source the grids at XY directions are stretched to provide smaller grid size of $R^{*}=1 / 20$.

\subsection{Results}

Figure 3 gives the 3D contour view of the distribution of gas temperatures in simulation. Figure 4 shows the distribution of gas temperatures at $X=0$. Figure 5 shows the comparison of the results for maximum plume center line temperatures predicted by correlations and FDS. Above the flame height calculated by Eq. 9, the gas temperatures predicted by FDS are higher than those by correlations; and below, the gas temperatures predicted by FDS are lower than those by correlations. The numerical results predicted by using fine grid sizes fit close to the results predicted by correlations. Figure 6 shows the comparison of the results for maximum ceiling jet temperatures predicted by correlations and FDS. The numerical results predicted by using fine grid size fit close to those predicted by Alpert's correlations.

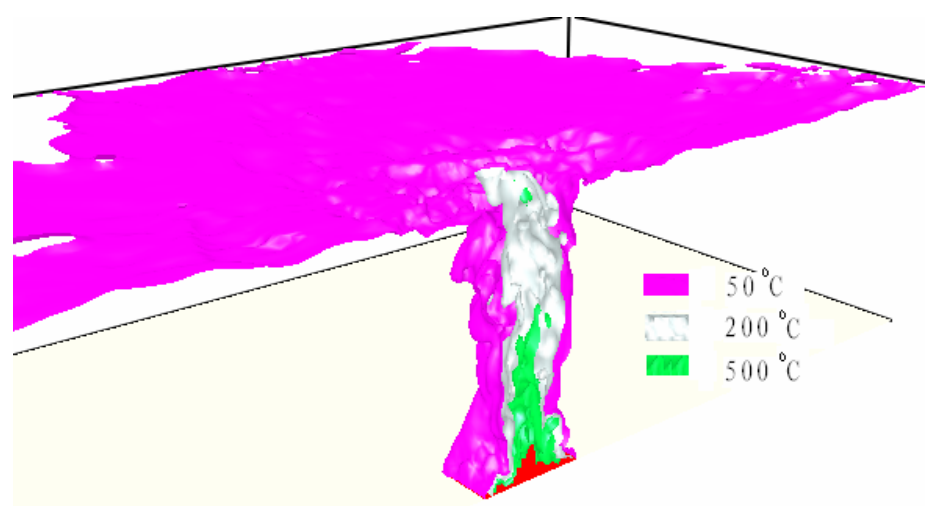

Figure 3. 3D Contour View of the Distribution of Gas Temperatures in the Design Fire Scenario

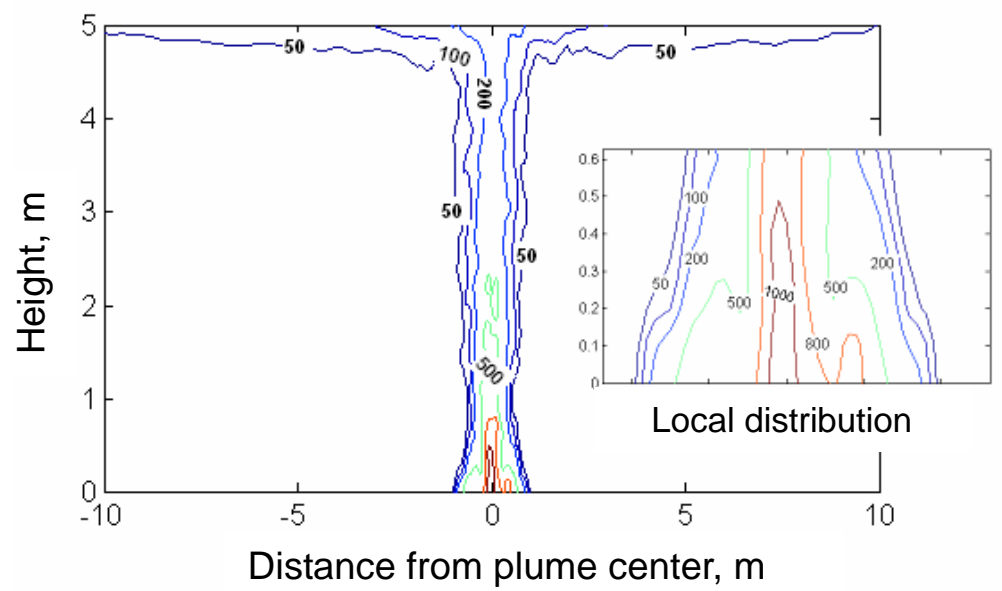

Figure 4. Numerical Results for the Distribution of Gas Temperatures in the Design Fire Scenario 


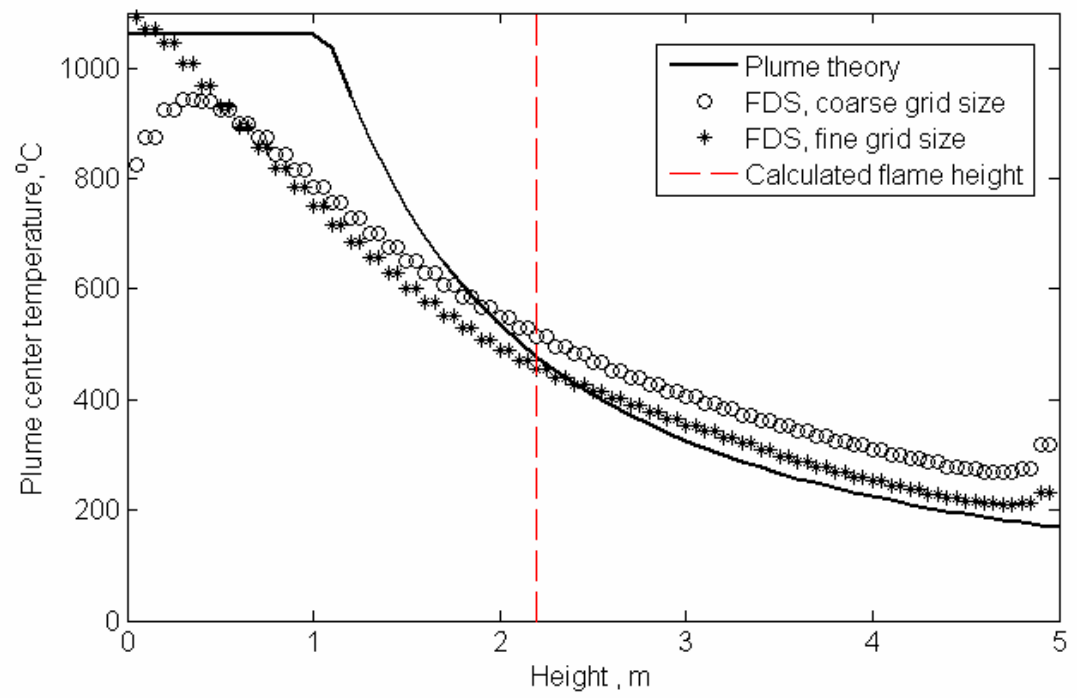

Figure 5. Results for Maximum Fire Plume Central Line Temperatures for the Design Fire Scenario

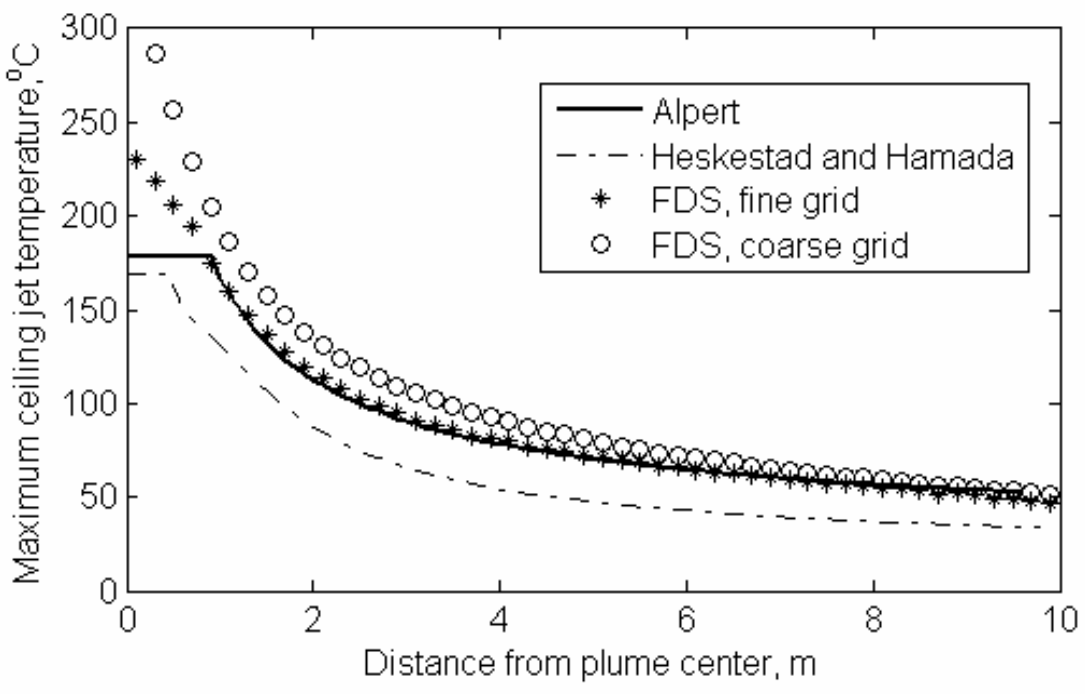

Figure 6. Results for Maximum Ceiling Jet Temperatures for the Design Fire Scenario

\section{SURROUNDING FIRE TETS}

\subsection{Description of the Experiment}

In Kamikawa et al.'s surrounding fire tests [7], a $2.50 \mathrm{~m}$ tall, $0.15 \mathrm{~m}$ square and $4.5 \mathrm{~mm}$ thick steel column were prepared as the specimen, as illustrated in Figure 7. Eight $0.15 \mathrm{~m}$ square porous burners with propane as the fuel were placed around the $0.15 \mathrm{~m}$ square column to make a $0.45 \mathrm{~m}$ square burner-column complex. The total heat release rate (HRR) was controlled by fuel supply rate to range from 40.5 to $162 \mathrm{~kW}$. Heat fluxes to the column surfaces were measured by $15 \mathrm{~mm}$ diameter Schmidt-Boelter heat flux gauges and temperatures of the column surfaces were measured by K-type thermocouples. 


\subsection{FDS Model}

Figure 7 shows the geometry of the numerical model and calculation mesh used in FDS simulation. The computational domain has dimensions of $0.6 \mathrm{~m}(\mathrm{x}) \times 0.6 \mathrm{~m}(\mathrm{y}) \times 2.4 \mathrm{~m}(\mathrm{z})$. The grids in all directions are uniform. For the burner area, the heat release rate per unit area (HRRPUA) was specified to generate the same HRR as in the experiments. OPEN vents were used on the exterior mesh boundaries to represent opening conditions in the experiments [7]. Thermal properties of the steel were taken from [21]. The default emissivity of solid surfaces (0.9) was used.
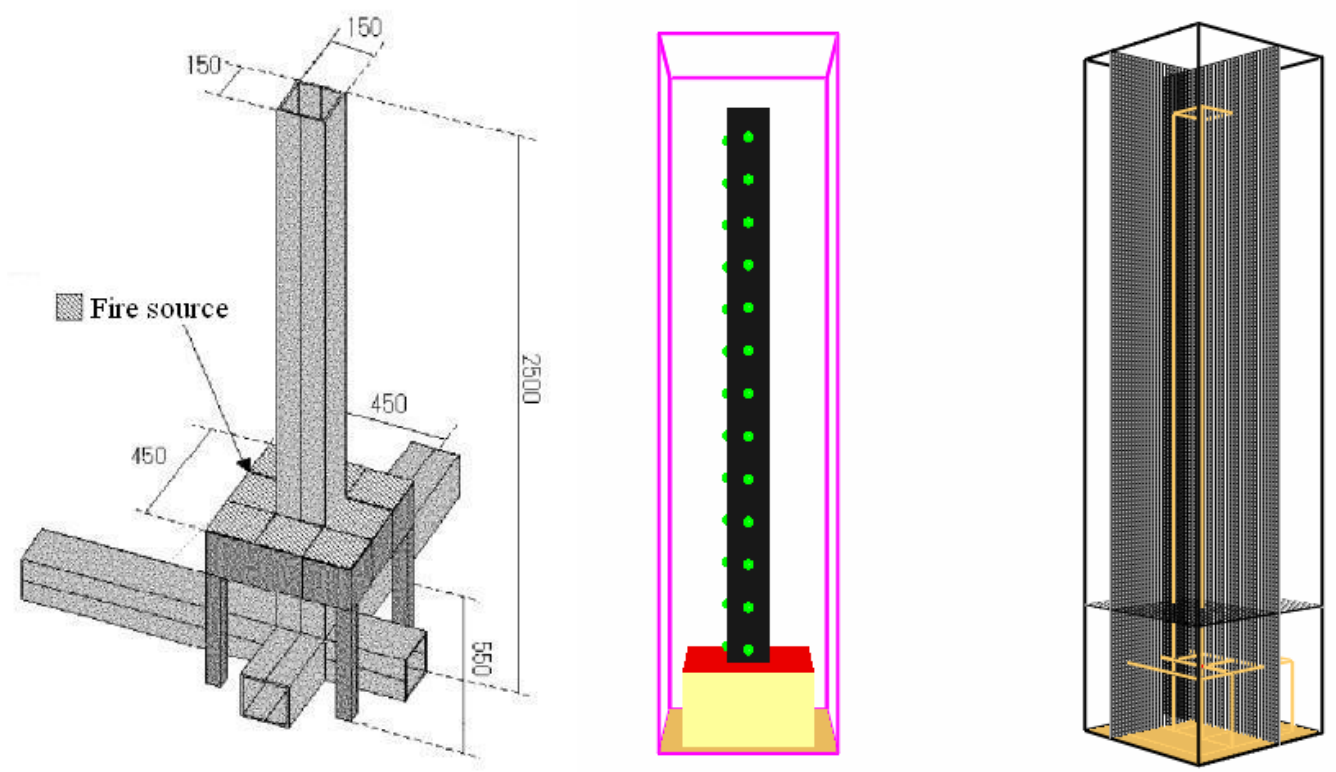

Figure 7. Experimental Setup in [7], the Corresponding Numerical Model and Calculation Mesh used in FDS Simulation

\subsection{Sensitivity Studies}

\subsubsection{Grid sizes}

Figure 8 shows the results of predicted heat fluxes using different resolution $R^{*}$, for $40.5 \mathrm{~kW}$ fire test. The height $z$ is the vertical distance from the measuring point to the burner surface. Resolutions ranged from 1/8.9 to $1 / 35.6$ are considered. It shows the predicted results using $R^{*}=1 / 8.9$ to $1 / 16.0$ are similar, also for $R^{*}=1 / 17.8$ to $1 / 28.6$. The predicted results using resolution of about $1 / 32.0$ fit very well with the test data.

Figure 9 shows the results of predicted heat fluxes using different resolution $R$, for $81 \mathrm{~kW}$ fire test. Resolutions ranged from 1/8.8 to $1 / 29.3$ are considered. It shows the predicted results using $R^{*}=1 / 11.7$ to $1 / 14.7$ are similar, also for $R^{*}=1 / 17.6$ to $1 / 21.1$, and $R^{*}=1 / 23.5$ to $1 / 29.3$. The predicted results using $R^{*}=1 / 23.5$ to $1 / 29.3$ fit very well with the test data.

Figure 10 shows the results of predicted heat fluxes using different resolution $R^{*}$, for $121.5 \mathrm{~kW}$ fire test. Resolutions ranged from $1 / 13.8$ to $1 / 31.1$ are considered. The predicted results using resolution of about $1 / 27.6$ fit well with the test data.

Figure 11 shows the results of predicted heat fluxes using different resolution $R^{*}$, for $162 \mathrm{~kW}$ fire test. Resolutions ranged from 1/19.4 to $1 / 34.8$ are considered. It shows the predicted results using $R^{*}=1 / 23.2$ to $1 / 34.8$ are similar. The predicted results using resolution using $R^{*}=1 / 23.2$ to $1 / 34.8$ fit well with the test data. 


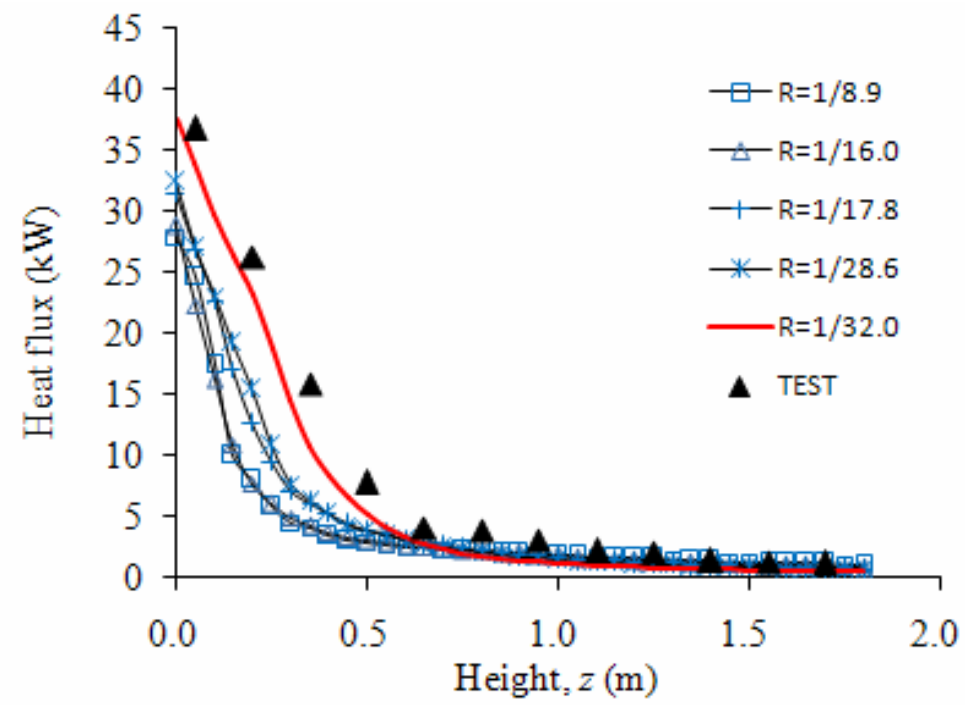

Figure 8. Results of Predicted Heat Fluxes Using Different R*, 40.5 kW

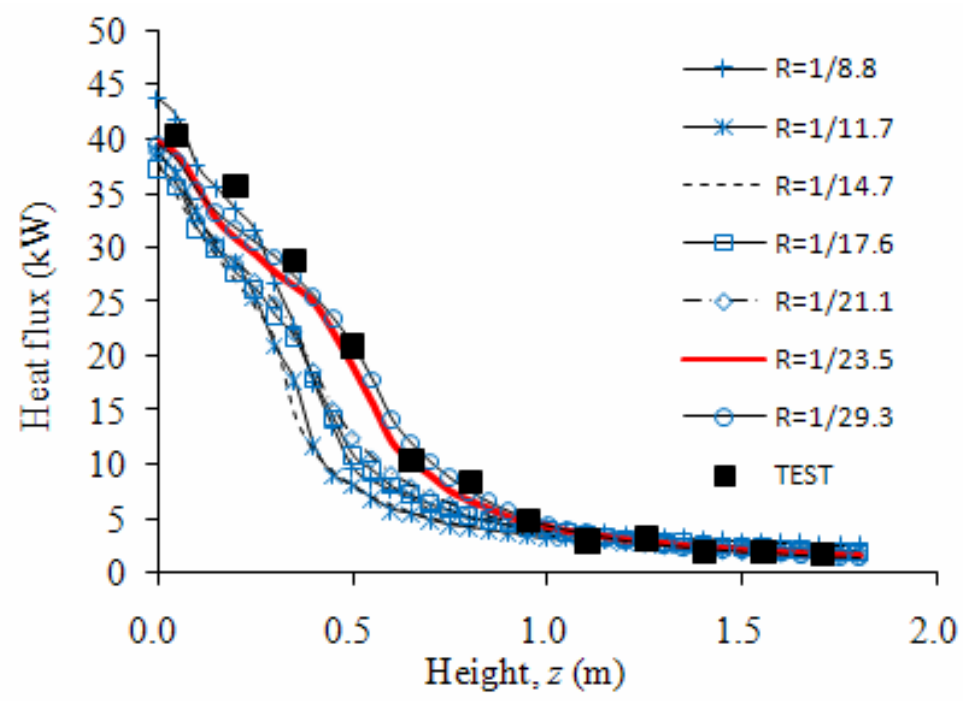

Figure 9. Results of Predicted Heat Fluxes Using Different R*, 81 kW

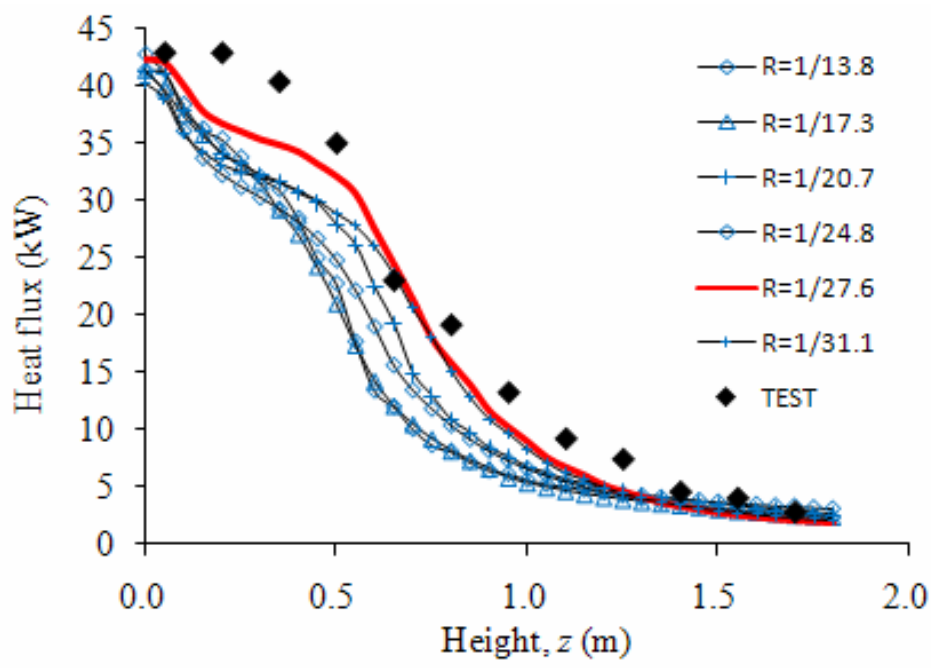

Figure 10. Results of Predicted Heat Fluxes Using Different R*, 121.5 kW 


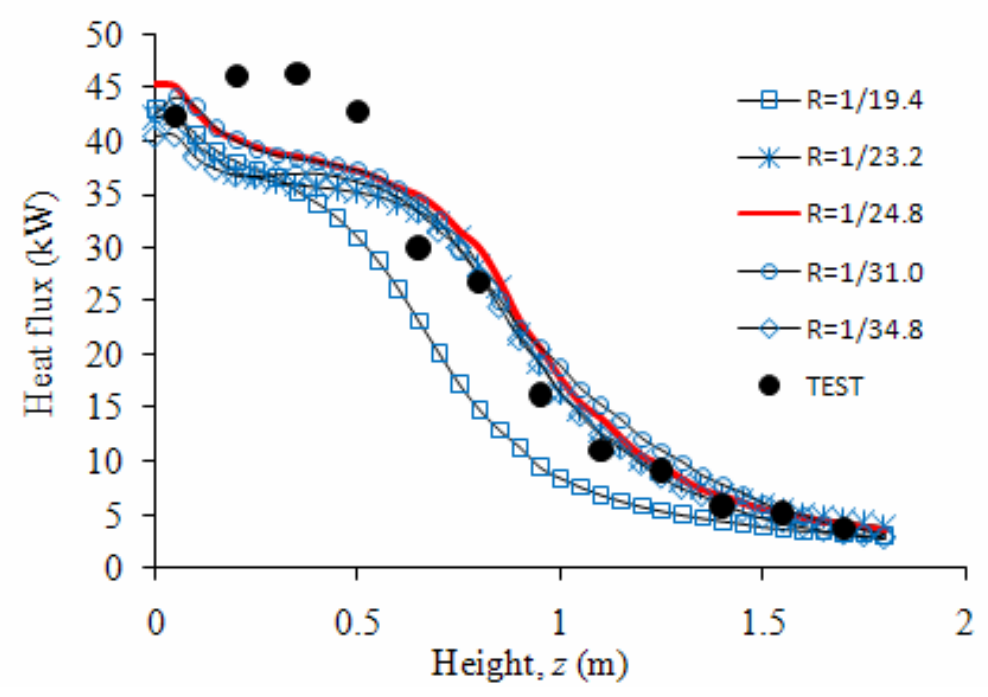

Figure 11. Results of Predicted Heat Fluxes Using Different R*, 162 kW

For all cases, using resolution of about $1 / 30$ can give good prediction of heat fluxes. Table 1 gives the grid sizes used in the following simulations.

Table 1. Simulation Inputs

\begin{tabular}{ccccccc}
\hline$H R R(\mathrm{~kW})$ & $D^{*}$ & Grid number & $\delta x$ & $R^{*}$ & $N S A$ & $\chi_{r}$ \\
\hline 40.5 & 0.2668 & $72 \times 72 \times 288$ & 0.0083 & $1 / 32.0$ & 300 & 0.35 \\
81 & 0.3521 & $40 \times 40 \times 160$ & 0.0150 & $1 / 23.5$ & 300 & 0.35 \\
121.5 & 0.4141 & $40 \times 40 \times 160$ & 0.0150 & $1 / 27.6$ & 300 & 0.35 \\
162 & 0.4646 & $32 \times 32 \times 128$ & 0.0188 & $1 / 24.8$ & 300 & 0.35 \\
\hline
\end{tabular}

\subsubsection{Number of solid angles}

Number of solid angles (NSA) is very significant for the accurate prediction of the radiative heat fluxes [22]. 104 solid angles is default in FDS. In Ref. [23], 304 solid angles were used for radiation solver to ensure accuracy of the FVM solution. Lin et al. [20] suggested 500 solid angles (when using gray model to solve RTEs ) are enough to predict the radiative heat fluxes emitted from the burners.

Figure 12 shows the distribution of predicted heat fluxes using different NSAs. It shows the numerical results are not sensitive to NSA, whilst using more solid angles needs more computing times. As a result, 300 solid angles is used in the simulations, as given in Table 1.

\subsection{Results and Discussions}

For HRRs of $40.5 \mathrm{~kW}$ and $81 \mathrm{~kW}$, the predicted heat fluxes agree very well with the test data. For HRRs of $121.5 \mathrm{~kW}$ and $162 \mathrm{~kW}$, overall the predicted heat fluxes agree very well with the test data, whereas at low elevation the predicted results divergence slightly to the test data.

Figures 12 and 13 show the comparisons between the predicted surface temperatures and the test data. For HRR of $81 \mathrm{~kW}$, the predicted surface temperatures agree very well with the test data. For HRR of $40.5 \mathrm{~kW}$, the predicted surface temperatures agree well with the test data. For HRRs of $121.5 \mathrm{~kW}$ and $162 \mathrm{~kW}$, overall the predicted temperatures agree very well with the test data, whereas at low elevation the predicted results divergence slightly to the test data. 


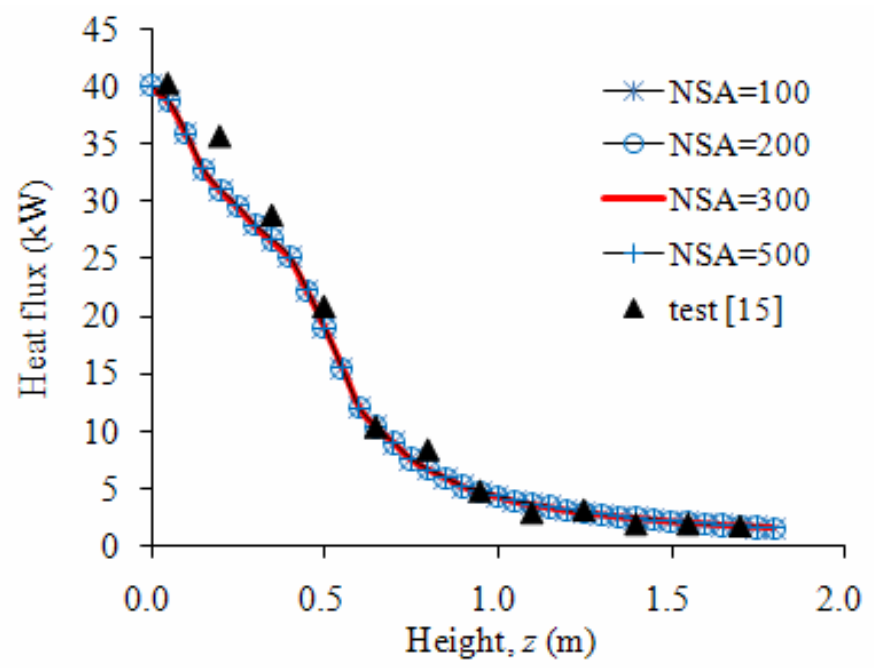

Figure 12. Distribution of Predicted Heat Fluxes Using Different NSAs

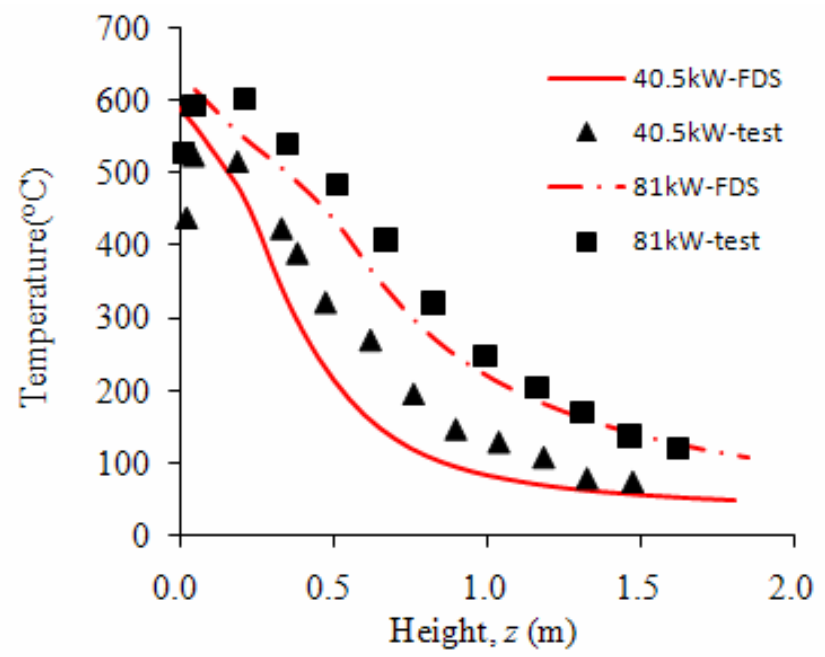

Figure 13. Comparisons of Predicted Surface Temperature with the Test Data for HRRs of $40.5 \mathrm{~kW}$ and $81 \mathrm{~kW}$

Those differences between the predicted and measured results could be mainly attributed to the accuracy of the sub-models in FDS. It is well known that the accuracy of combustion and radiation models has a paramount effect on the heat flux prediction. Although FDS provides a reliable flow solver, the mixture fraction combustion model could yield errors at large as 20 percent as suggested by the code developer [9] and the radiation model cannot solve accurately the radiation equation with grid sizes on the order of centimeter due to the fourth power dependence of radiation on temperature.

\section{CONCLUSIONS}

The application of using the popular CFD code FDS to predict the thermal actions in localized fires has been investigated. Based on the results of this study, the following conclusions can be drawn:

- The CFD code FDS can simulate the thermal actions in localized fires with acceptable accuracy. The predicted gas temperatures in the design fire scenario agree with those predicted by correlations; and the predicted heat fluxes and steel temperatures in the surrounding fire tests fit well with the test data. 
- The grid size used is the most important input parameter affecting the numerical results. Comparing with correlations, simulation using fine grid gives better prediction of gas temperatures than simulation using coarse grid. Resolution of 1/20 can give good prediction of gas temperatures in localized fires. For surrounding fire tests, sensitivity studies shows that resolutions of $1 / 32,1 / 23.5,1 / 27.6$ and $1 / 24.8$ give best prediction of the heat fluxes for HRRs of $40.5 \mathrm{~kW}, 81 \mathrm{~kW}, 121.5 \mathrm{~kW}$ and $162 \mathrm{~kW}$ respectively (whilst using resolution of about 1/30 give acceptable prediction of heat fluxes for all cases). Those resolutions are finer than the expected values from literature (1/20 [13], $1 / 13$ [20]). As a result, it is recommended that the user should take special care of the grid size and sensitivity studies should be always conducted when performing simulations.

- The number of solid angels has negligible effect on the numerical results.

\section{REFERENCES}

[1] Drysdale, D., “An Introduction to Fire Dynamics”, 2nd Edition, John Wiley and Sons, 1999.

[2] Li, G.Q. and Zhang, C., “Thermal Response to Fire of Uniformly Insulated Steel Members : Background and Verification of the Formulation Recommened by Chinese Code CECS200", Advanced Steel Construction, 2010, Vol. 6, pp. 788-802.

[3] Zhang, C., Li, G.Q. and Wang, Y.C., "Sensitivity Study on Using Different Formulae for Calculating the Temperature of Insulated Steel Members in Natural Fires”, Fire Technology, 20120, Vol. 48, pp.343-66.

[4] Li, G.Q. and Zhang, C., "Simple approach for calculating maximum temperature of insulated steel members in natural-fires", Journal of Constructional Steel Research, 2012, Vol.71, pp. 104-10.

[5] EN 1991-1-2, Eurocode 1: Actions on Structures - Part 1-2: General Rules - Actions on Structures Exposed to Fire, British Standard Institution, London, 2002.

[6] Brian, Y. Lattimer, "Heat Fluxes from Fires to Surfaces", SFPE Handbook 3rd Edition, Sec. 2-14, National Fire Protection Association, 2003.

[7] Kamikawa, D., Hasemi, Y., Wakamatu, T. and Kagiya, K., "Experimental Flame Heat Transfer and Surface Temperature Correlations for a Steel Column Adjacent to and Surrounded by a Pool Fire”, Proceedings of IFASS 2002, 2002.

[8] Li, G.Q. and Zhang, C., "Thermal Response of Steel Columns Exposed to Localized Fires Numerical Simulation and Comparison with Experimental Results”, Proceedings of the Sixth International Conference on Structures in Fire, MI, USA, 2010, pp. 35-42.

[9] McGrattan, K., McDermott, R., Hostikka, S. and Floyd, J., "Fire Dynamics Simulator (Version 5) User's Guide”, NIST Special Publication 1019-5, National Institute of Standards and Technology (NIST), Gaithersburg, Maryland; 2010.

[10] Quintiere, J.G., “Fundamentals of Fire Phenomena”, John Wiley \& Sons, Ltd., 2006.

[11] Heskestad, G., "Fire Plumes, Flame Height, and Air Entrainment”, SFPE Handbook 3rd Edition, Sec. 2-1, National Fire Protection Association, 2003.

[12] Quintiere, J.G. and Grove, B.S., “A Unified Analysis for Fire Plumes”, 27 ${ }^{\text {th }}$ Symposium (International) on Combustion”, The Combustion Institute, 1998, pp. 2757-66.

[13] Ma, T.G. and Quintiere, J.G., "Numerical Simulation of Axi-symmetric Fire Plumes: Accuracy and Limitations”, Fire Safety Journal, 2003, Vol. 38, pp. 467-92.

[14] Alpert, R., "Calculation of Response Time of Ceiling-mounted Fire Detectors". Fire Technology, 1972, Vol. 8, pp. 181-95.

[15] PD 7974-2, Application of Fire Safety Engineering Principles to the Design of Buildings Part 2: Spread of Smoke and Toxic Gases within and Beyond the Enclosure of Origin (Sub-system 2), British Standard Institution, London, 2002. 
[16] Heskestad, G. and Hamada, T., "Ceiling Jets of Strong Fire Plumes”, Fire Safety Journal, 1993, Vol. 21, pp. 69-82.

[17] Grosshandler, W.L., "RADCAL: A Narrow-band Model for Radiation Calculations in a Combustion Environment”, National Institute of Standard and Technology, NIST Technical Note 1402, 1993.

[18] Chow, W.K., Li, Y.Z., Cui, E. and Huo, R., "Natural Smoke Filling in Atrium with Liquid Pool Fires Up to 1.6MW”, Building and Environment, 2001, Vol. 36, pp. 121-27.

[19] Gutierrez-Montes, C., Sanmiguel-Rojas, E., Viedma, A. and Rein, G., "Experimental Data and Numerical Modeling of 1.3 and 2.3 MW Fires in a $20 \mathrm{~m}$ Cubic Atrium,” Building and Environment, 2009, Vol. 44, pp. 1827-39.

[20] Lin, C.H., Ferng, Y.M, and Hsu, W.S., "Investigating the Effect of Computational Grid Sizes on the Predicted Characteristics of Thermal Radiation for a Fire", Applied Thermal Engineering, 2009, Vol. 29, pp. 2243-50.

[21] Wakamatsu, T., Hasemi, Y. and Ptchelintsev, A.V., "Heating Mechanism of Building Components Exposed to a Localizedfire: CFD Prediction of the Heat Flux of a Steel Beam”, Fire Science and Technology, 2000, Vol. 20, pp. 1-12.

[22] Jensen, K.A., Ripoll, J-F., Wray, A.A., Joseph, D. and Mouna EI Hafi, “On Various Modeling approaches to Radiative Heat Transfer in Pool Fires”, Combustion and Flame, 2007, Vol. 148, pp. 263-79.

[23] Hostikka, S., McGrattan, K. and Hamins, A., "Numerical Mdeling of Pool Fires Using LES and Finite Volume Method for radiation. Fire Safety Science - Proceedings of the 7th International symposium, pp. 383-94. 